\title{
The effect of COVID-19 stay-at-home order and campus closure on the prevalence of acute respiratory infection symptoms in college
} campus cohorts.

\author{
Oluwasanmi Adenaiye ${ }^{1}$, Paul Jacob Bueno de Mesquita ${ }^{1}$, Qiong $\mathrm{Wu}^{1}$, Filbert Hong ${ }^{1}$, \\ Jianyu Lai ${ }^{1}$, Shuo Chen ${ }^{2}$, and Donald Milton ${ }^{1}$ \\ ${ }^{1}$ University of Maryland at College Park \\ ${ }^{2}$ University of Maryland Baltimore
}

November 2, 2020

\begin{abstract}
Evaluation of population-based COVID-19 control measures informs strategies to quell the current pandemic and reduce the impact of those yet to come. Effective COVID-19 control measures may simultaneously reduce the incidence of other acute respiratory infections (ARIs) due to shared transmission modalities. To assess the impact of stay-at-home orders and other physical distancing measures on the prevalence of ARI-related symptoms, we compared symptoms reported by prospective college cohorts enrolled during two consecutive academic years. ARI-related symptoms declined following campus closure and implementation of stay-at-home orders, demonstrating the impact of population-based physical distancing measures on control of a broad range of respiratory infections.
\end{abstract}

\section{Hosted file}

The effect of COVID-19 stay-at-home orders and campus closure on the prevalence of ARI-related symptoms available at https://authorea.com/users/372146/articles/490289-the-effect-of-covid-19stay-at-home-order-and-campus-closure-on-the-prevalence-of-acute-respiratory-infectionsymptoms-in-college-campus-cohorts 